\title{
Using Polarimetry To Determine The CEBAF Beam Energy
}

\section{Douglas W. Higinbotham*}

Jefferson Lab

E-mail: doug@jlab.org

\begin{abstract}
As Jefferson Lab begins operations with its upgraded CEBAF accelerator, the lab once again needs to experimentally determine the absolute beam energy of the machine. Previously, the CEBAF beam energy was determined using precision measurements of the bending magnet integral fields along with beam position information. The result obtained from this technique was crosschecked with elastic scattering from hydrogen where knowledge of the scattering angles of the electron and proton allow the beam energy to be determined. While the field integral method will still work with the upgraded machine, the elastic cross section becomes too small to make precision measurements at angles that are large enough to be easily accessible; thus a new technique for energy determination has been sought. It will be shown that by making use of polarimetry, one can use CEBAF's polarized electrons' $\mathrm{g}$-2 spin precession to determine the absolute beam energy. This can be done in a single hall if the parameters of the machine, such as the injector energy and linac imbalance, are known or with two halls without any knowledge of the machine parameters.
\end{abstract}

XVth International Workshop on Polarized Sources, Targets, and Polarimetry

September 9 - 13, 2013

Charlottesville, Virginia, USA

\footnotetext{
* Speaker.
} 


\section{Introduction}

Absolute determination of Jefferson Lab's CEBAF beam energy is required by the nuclear physics experiments carried out in the lab's experimental halls. During the $6 \mathrm{GeV}$ era at the lab, two techniques were used to determine the beam energy. The first made use of well calibrated dipole magnets along with beam position information and the second used precise measurements of elastic proton-electron scattering angles. Both of these measurements were able to determine the absolute beam energy of CEBAF to the $10^{-4}$ level.

With the increase in the Jefferson Lab beam energy to $11 \mathrm{GeV}$ [1], the ability to make the elastic proton-electron scattering angle measurements gets extremely challenging due to the small cross sections at reasonably large angles. Due to this challenge, a new high precision technique for determining the beam energy was sought.

\section{Spin Precession}

During the $6 \mathrm{GeV}$ era, it was experimentally demonstrated that by making use of the polarized electron beam in conjunction with high precision polarimetry, one could determine the beam energy

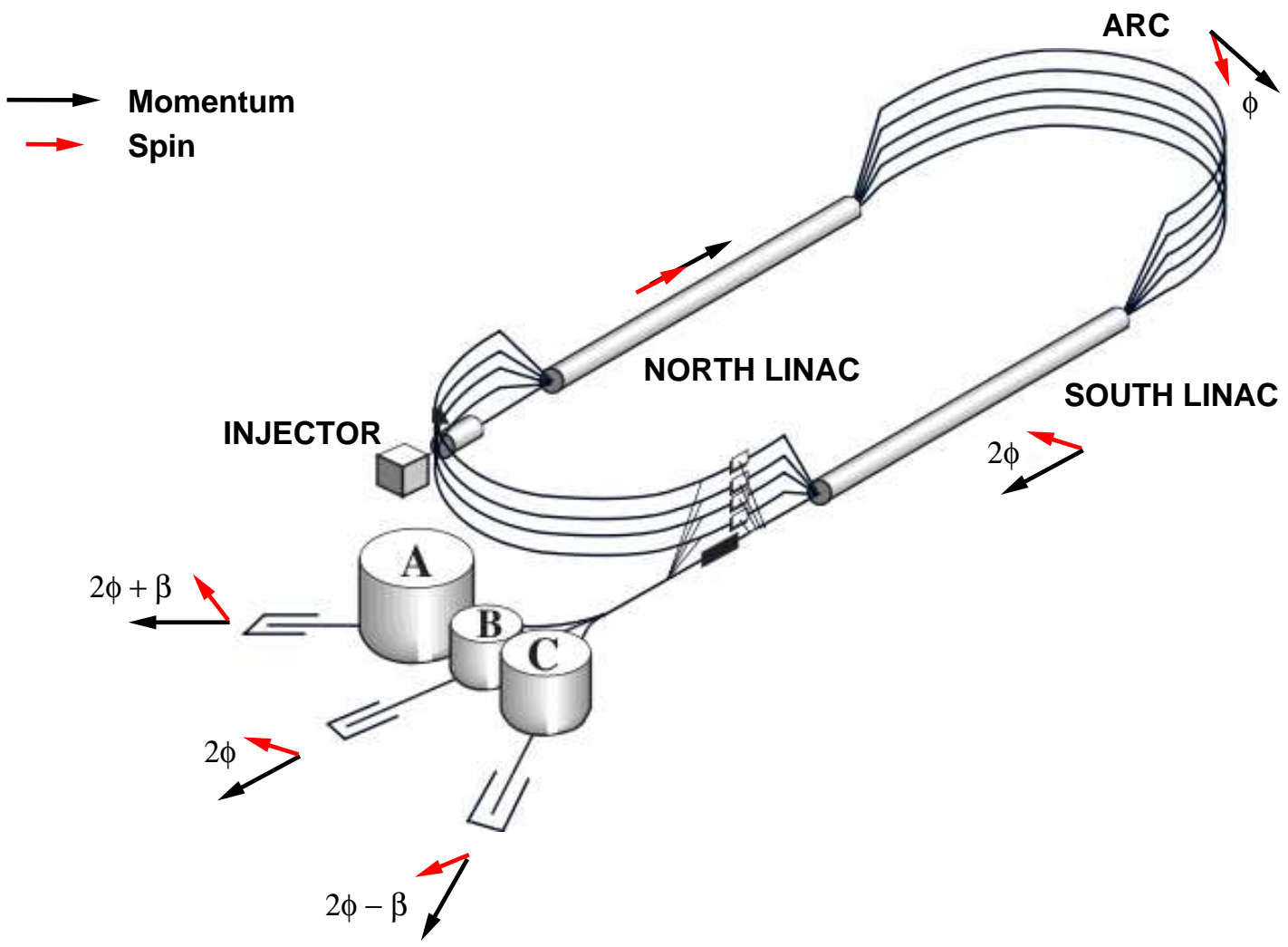

Figure 1: Shown is an example of spin precession through the CEBAF accelerator. In the example, the momentum and spin vectors point in the same direction at the injector; but due to the electron's anomalous magnetic moment, those vectors will start to point in different directions as the electrons are bent through the arc magnets and into the halls. 
at nearly the same level as the other two techniques [2]. This was done by determining the Wien angle that gave the maximum longitudinal polarization to each hall for a given set of accelerator parameters. Interestingly, while the ability to do the elastic cross section measurements gets harder as the beam energy increases, the rate of spin precession increases with the increasing energy and makes it possible to do even more precise energy determinations using exactly the same polarimetry equipment as previously used.

The spin precession, $\Delta \Phi$, of an electron as it bends through a series of dipole magnets is given by:

$$
\Delta \phi=\frac{\mathrm{E}}{440.65 \mathrm{MeV}} \times \Delta \theta
$$

where $E$ is the energy of the electron and $\Delta \theta$ is the bend angle [3]. Figure 1 shows an example of this type of precession for a polarized electron leaving the CEBAF injector and being sent one time around the machine and then split into the three Halls. In typical operations, the beam goes around the machine several times, and since the precession increases with each pass through the linear accelerators, total precession can get be thousands of degrees before the beam reaches an experimental hall.

\section{Types of Spin Measurements}

The energy of the beam can be determined with two types of spin measurement. In one case, you make use of the knowledge of the accelerator parameters such as the injector Wien angle, injector energy and linac balance along with polarimetry in one hall. In the other, you simply make polarimetry measurements in two halls. For this type of measurement, only the bend angle between the two halls, $\Delta \Theta$, and the difference in the Wien angle that provides maximum polarization to each hall, $\Delta \Phi$, given by:

$$
\Delta \Phi=\frac{\mathrm{E}}{440.65 \mathrm{MeV}} \times \Delta \Theta,
$$

is needed. This latter technique is not as precise as the first as the precession is only measured from the beam switch yard instead of the full precession through the machine.

\section{Systematic Errors}

With many thanks to the Department of Energy's SULI program, summer student Gina Mayonado was able to write an Apple application to calculate the spin precession through the CEBAF accelerator and study the effects of various systematic uncertainties. For this study, the following parameters were used: injector Wien angle known to one degree and linac energy difference less then two MeV. The $6 \mathrm{GeV}$ CEBAF accelerator not only had these parameters under control at this level, changing them at this level was used to increase the magnitude of simultaneous, longitudinal polarization to the experimental halls [4]. Table 4 shows the systematic beam energy determination uncertainty for difference passes for a CEBAF acceleration of $2.2 \mathrm{GeV}$ per pass. For the two hall case, the ability to determine the beam energy is closer to $10^{-3}$ simply because of the very limited total precession between the beam switch yard and the halls as compared to the precession through the entire machine. 


\begin{tabular}{cccc} 
Number of Passes & $\begin{array}{c}\text { Hall A } \\
\mathrm{MeV}\end{array}$ & $\begin{array}{c}\text { Hall B } \\
\mathrm{MeV}\end{array}$ & $\begin{array}{c}\text { Hall C } \\
\mathrm{MeV}\end{array}$ \\
\hline 1 & 3.80 & 5.38 & 9.23 \\
2 & 2.05 & 2.33 & 2.71 \\
3 & 1.70 & 1.84 & 2.01 \\
4 & 1.61 & 1.69 & 1.80 \\
5 & 1.81 & 1.83 & 1.94
\end{tabular}

Table 1: Shown is the total systematic uncertainty for single hall determination of the beam energy where it has been assumed that the injector Wien angle is known absolutely to one degree, the difference in linac energies is less then two $\mathrm{MeV}$, and that the effect of synchrotron radiation can be accounted for to the 3\% level; though the synchrotron correction is only important for the 5 th pass.

\section{Conclusions}

With the upgraded CEBAF accelerator, a new series of energy measurements will be preformed. By making use of the classic integral field measurements along with spin precession measurements; the energy of the upgraded machine will be determined with independent techniques both of which are capable of achieving few $10^{-4}$ level precision.

\section{References}

[1] J. Grames, D. W. Higinbotham and H. E. Montgomery, "Thomas Jefferson National Accelerator Facility," Nucl. Phys. News 20N3, 6 (2010).

[2] J. M. Grames, C. K. Sinclair, J. Mitchell, E. Chudakov, H. C. Fenker, A. Freyberger,

D. W. Higinbotham and M. Poelker et al., "Unique electron polarimeter analyzing power comparison and precision spin-based energy measurement," Phys. Rev. ST Accel. Beams 7, 042802 (2004) [Erratum-ibid. 13, 069901 (2010)].

[3] B. W. Montague, "Polarized Beams in High-energy $e^{+} e^{-}$Storage Rings," Phys. Rept. 113, 1 (1984).

[4] D. W. Higinbotham, "Electron Spin Precession at CEBAF,” AIP Conf. Proc. 1149, 751 (2009) [arXiv:0901.4484 [physics.acc-ph]]. 